\title{
An improvement of the BeppoSAX LECS and MECS positioning accuracy
}

\author{
M. Perri ${ }^{1,2}$ and M. Capalbi ${ }^{1}$ \\ 1 ASI Science Data Center, c/o ESA-ESRIN, via Galileo Galilei, 00044 Frascati, Italy \\ e-mail: perri@asdc.asi.it, capalbi@asdc.asi.it \\ 2 Dipartimento di Fisica, Università "La Sapienza”, Piazzale A. Moro 2, 00185 Roma, Italy
}

Received 5 August 2002 / Accepted 3 October 2002

\begin{abstract}
We present a study of the source positioning accuracy of the LECS and MECS instruments on-board BeppoSAX. From the analysis of a sample of archival images we find that a systematic error, which depends on the spacecraft roll angle and has an amplitude of $\sim 17^{\prime \prime}$ for the LECS and $\sim 27^{\prime \prime}$ for the MECS, affects the sky coordinates derived from both instruments.

The error is due to a residual misalignment of the two instruments with respect to the spacecraft $Z$ axis arisen from the presence of attitude inaccuracies in the observations used to calibrate the pointing direction of LECS and MECS optical axes.

Analytical formulae to correct LECS and MECS sky coordinates are derived. After the coordinate correction the $90 \%$ confidence level error radii are 16" and 17" for LECS and MECS respectively, improving by a factor of $\sim 2$ the source location accuracy of the two instruments. The positioning accuracy improvement presented here can significantly enhance the follow-up studies at other wavelengths of the X-ray sources observed with LECS and MECS instruments.
\end{abstract}

Key words. instrumentation: miscellaneous - X-rays: general

\section{Introduction}

The 0.1-10 keV imaging instruments on-board the BeppoSAX satellite (Boella et al. 1997a) contribute significantly to the study of the X-ray sky.

Among the important topics studied, we can certainly include the investigation of the nature of the sources producing the Cosmic X-ray Background (Giommi et al. 1998, 2000; Fiore et al. 1999, 2001; Comastri et al. 2001; Vignali et al. 2001; La Franca et al. 2002), the study of the X-ray afterglows of Gamma-Ray Bursts (e.g., Antonelli et al. 2000; Feroci et al. 2001; in 't Zand et al. 2001; Piro et al. 2002) and the identification of new X-ray transients in the galactic bulge (e.g., the galactic black hole candidate XTE J1908+094, in 't Zand et al. 2002).

Crucial, in all these studies, is an accurate determination of the celestial coordinates of the imaged X-ray source, since it may allow a firm identification of its counterpart at other wavelengths.

Previous discussions of the positioning accuracy of the BeppoSAX MECS instrument (Ricci et al. 1998; Fiore et al. 2001) reported an error of $\sim 1$ arcmin at the $90 \%$ confidence level and pointed out that a significant contribution to the X-ray coordinate error comes from systematic uncertainties due to the absolute spacecraft attitude reconstruction.

Send offprint requests to: M. Perri, e-mail: perri@asdc . asi .it
With the aim of understanding the origin of these systematic uncertainties we present here a detailed study of the source location accuracy of the BeppoSAX imaging instruments.

\section{Data analysis}

The scientific instrumentation on-board the BeppoSAX satellite includes four co-aligned X-ray telescopes, each composed of a grazing incidence Mirror Unit and of a position sensitive Gas Scintillation Proportional Counter located at the focal plane.

Three of these systems are nearly identical and, collectively, constitute the Medium Energy Concentrator Spectrometer (MECS, Boella et al. 1997b). The three units, named MECS1, MECS2 and MECS3, are sensitive in the 1.3-10 keV energy band.

The fourth system, the Low Energy Concentrator Spectrometer (LECS, Parmar et al. 1997), has a mirror design identical to the one of the other three units, but the detector is sensitive to X-ray photons in the $0.1-10 \mathrm{keV}$ energy band.

The image size of both instruments is $256 \times 256$ pixels and the detector pixel size, near the center of the field of view, is $\sim 14^{\prime \prime}$ for the LECS and $\sim 19^{\prime \prime}$ for the MECS.

We have studied the source positioning accuracy of LECS and MECS instruments using a set of X-ray sources observed 

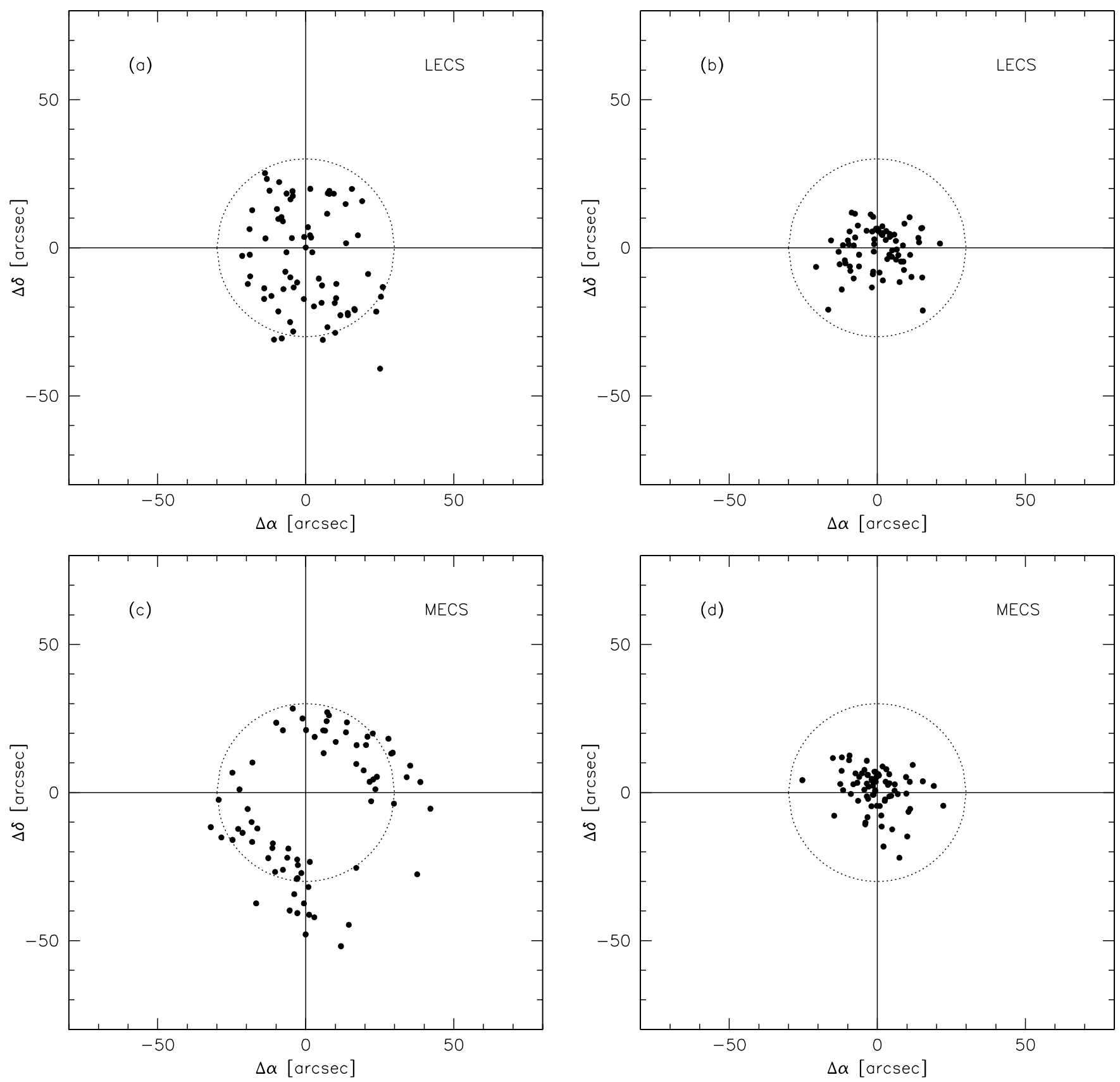

Fig. 1. a) The deviation in RA and Dec between LECS and optical positions for observations performed after May 6th 1998. b) The same plot after the coordinate correction discussed in the text. c) The deviation in RA and Dec between MECS and optical positions for observations performed after May 6th 1998. d) The same plot after the coordinate correction discussed in the text. The dotted circles have a radius of $30^{\prime \prime}$.

by BeppoSAX for which the position of the optical counterpart is known to within $1^{\prime \prime}$.

LECS and MECS fields have been selected according to the following criteria:

- the target is on-axis and relatively bright $\left(>10^{-2}\right.$ cts s $^{-1}$ for both LECS and MECS)

- the target is neither extended nor confused

- the whole range of spacecraft roll angle values, from $-90^{\circ}$ to $+270^{\circ}$, is uniformly covered (for a definition of roll angle see the Appendix)

- the observation has been performed later than May 6th 1998.
The last condition has been imposed to minimize position uncertainties since the BeppoSAX attitude data before May 6th 1998 are affected by relatively large inaccuracies (see Sect. 5).

The above conditions resulted in the selection of a sample of 72 X-ray fields which consists of 49 pointings of AGNs, 14 of Stars and 9 of X-ray binaries ${ }^{1}$.

LECS and co-added MECS2+MECS3 (hereafter MECS) images have been used. MECS1 data are not included in the analysis since this unit failed on May 6th 1997.

\footnotetext{
1 A table containing the list of the sources used in this analysis is available at http://www.asdc.asi.it/bepposax/positions/ table.html
} 
Table 1. LECS and MECS (2 units) $68 \%$ and $90 \%$ error radius values before and after the coordinate correction of Eqs. (7) and (8) for observations performed after May 6th 1998.

\begin{tabular}{cccc}
\hline \hline & Instrument & $68 \%$ radius & $90 \%$ radius \\
\hline before & LECS & $23^{\prime \prime}$ & $29^{\prime \prime}$ \\
correction & MECS & $30^{\prime \prime}$ & $41^{\prime \prime}$ \\
\hline after & LECS & $12^{\prime \prime}$ & $16^{\prime \prime}$ \\
correction & MECS & $11^{\prime \prime}$ & $17^{\prime \prime}$ \\
\hline
\end{tabular}

Images were accumulated in the $2-10 \mathrm{keV}$ band (channels 44-220) for the MECS and in the 0.1-9.5 keV band (channels 10-950) for the LECS. Event files from the BeppoSAX public archive (Giommi \& Fiore 1997) at the ASI Science Data Center (ASDC) have been used.

To determine the celestial coordinates of the X-ray sources we used the sky positions stored in the headers of LECS and MECS event files. The source detection has been performed using a variation of the DETECT routine of the XIMAGE package (Giommi et al. 1992) as described in Fiore et al. (2001).

Special attention has been devoted to the quality of the detections and each source position has been visually checked.

\section{Comparison with optical coordinates}

We have compared the celestial coordinates of the X-ray sources derived from LECS and MECS images with the accurate positions obtained from optical catalogs (Veron-Cetty \& Veron 1996; Turon et al. 1992; van Paradijs 1995).

We have then computed RA and Dec offsets between the optical and LECS/MECS values (labelled respectively "L" and "M") using the following definitions:

$\Delta \alpha_{\mathrm{L}, \mathrm{M}}=\left(\alpha_{\mathrm{opt}}-\alpha_{\mathrm{L}, \mathrm{M}}\right) \cos \delta$

$\Delta \delta_{\mathrm{L}, \mathrm{M}}=\delta_{\mathrm{opt}}-\delta_{\mathrm{L}, \mathrm{M}}$.

Note that RA offsets have been corrected by the factor $\cos \delta$ and represent therefore the true separation in the sky.

In Fig. 1 (panels a and c) we plot the measured $\Delta \alpha$ vs. $\Delta \delta$ values for the LECS and MECS instruments.

We have next computed the angular distances between the X-ray and optical positions. In Table 1 (top) we list the "radius" within which $68 \%$ and $90 \%$ of the objects are included. All the values in Table 1 have been rounded to unity.

\section{The dependence on roll angle}

As can be seen in Fig. 1 (panel c) the $\Delta \alpha$ and $\Delta \delta$ values for the MECS are not uniformly distributed around the zero values, indicating that a systematic error affects the source positioning accuracy of the instrument. For the LECS (panel a) we note that the same effect is not evident.

Moreover, Table 1 shows that the positions derived from the LECS instrument are more accurate (29" error radius at the $90 \%$ confidence level) with respect to those obtained from the $\operatorname{MECS}\left(41^{\prime \prime}\right)$.
To investigate in more details these results we have searched for a possible dependence of RA and Dec offsets on the satellite roll angle $\rho$.

In Fig. 2 we plot $\Delta \alpha$ and $\Delta \delta$ as a function of the spacecraft roll angle. As can be clearly seen, for both instruments a strong correlation between these quantities and the roll angle is found. Furthermore, we see that $\Delta \alpha$ and $\Delta \delta$ values follow a sinusoidal law and that the amplitude of this effect is $\sim 15^{\prime \prime}$ in the case of the LECS and $\sim 25^{\prime \prime}$ for the MECS.

We have also studied individually the single MECS units to verify if a specific offset dependence on the roll angle is present. We performed the analysis described above on MECS2 and MECS3 images separately obtaining the same results found in the study of the co-added images ${ }^{2}$.

The fact that LECS and MECS coordinate offsets are correlated with the satellite roll angle is a sure indication of a residual misalignment of both instruments with respect to the spacecraft $Z$ axis (the one co-aligned with the instruments).

The origin of the residual misalignment of LECS and MECS instruments is discussed in the next section.

\section{The LECS and MECS residual misalignment}

As already mentioned in Sect. 2, the BeppoSAX attitude data during the first two years of the mission (June 1996-May 1998) have been affected by relatively large systematic inaccuracies.

Since, as we will see, these inaccuracies are at the origin of the observed LECS and MECS residual misalignment, we briefly discuss this topic. Some useful definitions and formulae concerning the attitude can be found in the Appendix.

In summer 1997 an error of about 20" in the misalignment matrices of the three satellite star-trackers, used to track the guide stars and control the spacecraft attitude, was discovered.

As the following investigation revealed, the error originated because the coordinates of the guide stars used by the spacecraft star-trackers were not corrected for the annual aberration due to the heliocentric motion of the Earth.

A new computation of the star-trackers misalignment matrices was soon performed (August 1997), but during the following months rather large movements of the spacecraft (up to $\sim 2$ arcmin) when the attitude control was switched between different star-trackers were observed.

A detailed analysis of the problem pointed out an error in the correction for annual aberration of the guide stars coordinates which caused even larger errors in the star-trackers misalignment matrices and, consequently, larger attitude inaccuracies. A new and exact computation of the star-trackers misalignment matrices was then performed on May 6th 1998.

The problems listed above have determined spacecraft attitude data inaccuracies for observations carried out in the period June 1996-May 6th 1998. Due to both technical and financial reasons, a new computation of the attitude of this set of observations is not foreseen.

\footnotetext{
2 An analysis of the positioning accuracy of the single MECS units can be found at http://www.asdc.asi.it/bepposax/report/ report.html
} 

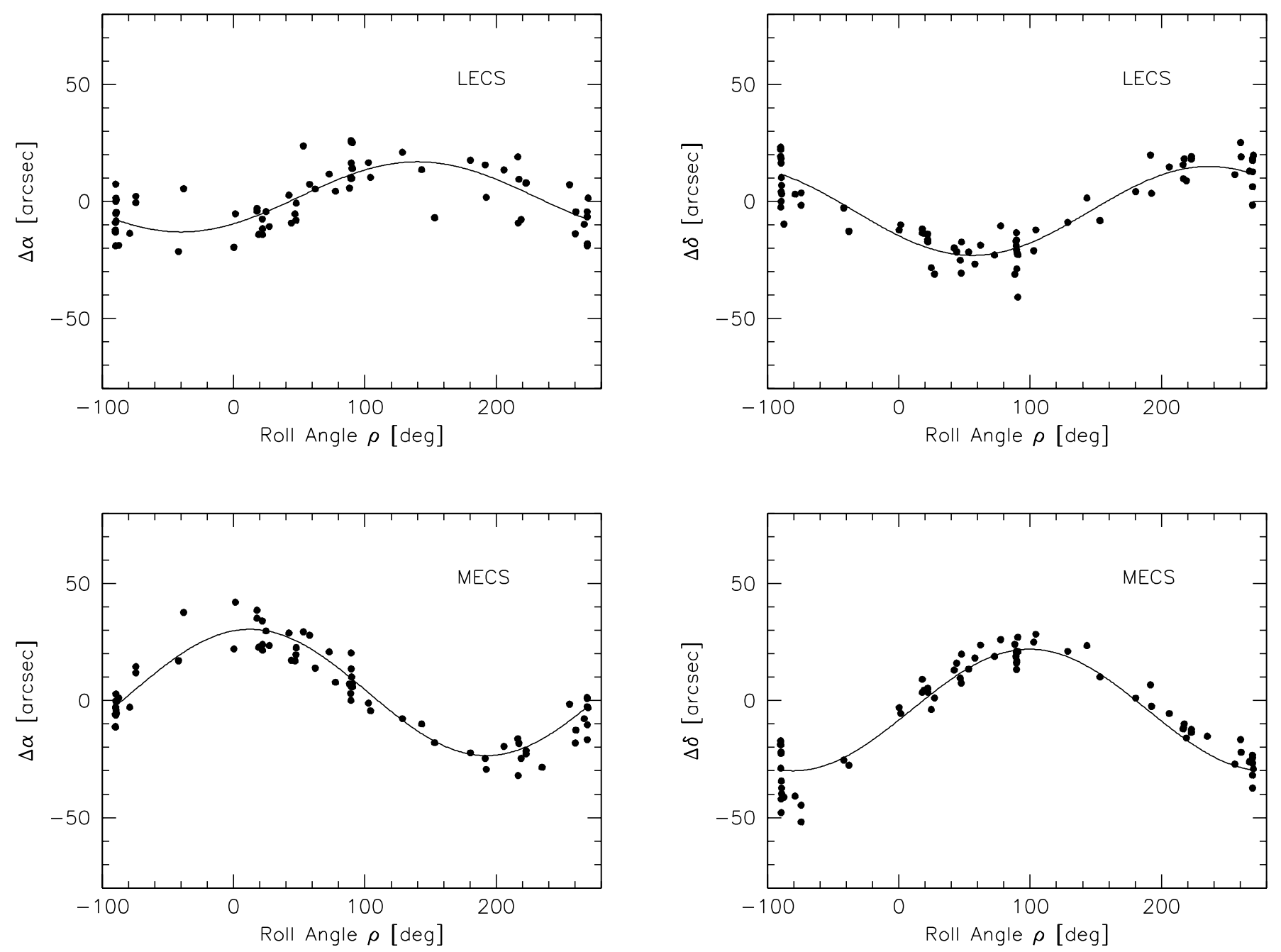

Fig. 2. Top: the differences in RA and Dec between LECS and optical positions plotted as a function of the spacecraft roll angle. Bottom: same plot for the MECS instrument. The solid lines are best fits to the data and are discussed in the text.

We identify these attitude inaccuracies as the cause of the observed residual misalignment of LECS and MECS instruments, as suggested by the following considerations. The misalignment of the X-ray telescopes with respect to the spacecraft $Z$ axis was accurately calibrated using a set of dedicated observations (Matteuzzi 1998) during the BeppoSAX Science Verification Phase. In the case of the MECS, the errors in the angles of the computed misalignment matrices $M_{\text {mis }}$ (see the Appendix for details) are $\sim 15^{\prime \prime}$ (Chiappetti, priv. comm.).

Since this set of observations was carried out in 1996, the calibration of LECS and MECS misalignment has been based on attitude data affected by significant inaccuracies. Our conclusion is that this fact has induced a systematic error in the misalignment matrices computation of the two instruments.

\section{Correction of LECS and MECS coordinates}

The observed dependence of LECS and MECS $\Delta \alpha$ and $\Delta \delta$ values on the spacecraft roll angle $\rho$ (see Fig. 2) allows us to parameterize, as a function of the roll angle, the LECS and MECS residual misalignment and to compute analytical formulae that can be used to correct the sky coordinates derived from the two instruments.

To this end, we have fitted the data plotted in Fig. 2 with the function $f(\rho)=A \cos (\rho+\phi)+c$. We obtained the following best fits for LECS and MECS respectively:

$$
\begin{array}{cc}
\Delta \alpha_{\mathrm{L}}=15 \cos (\rho-140)+2 & {[\operatorname{arcsec}]} \\
\Delta \delta_{\mathrm{L}}=19 \cos (\rho-236)-4 & {[\operatorname{arcsec}]} \\
\Delta \alpha_{\mathrm{M}}=27 \cos (\rho-12)+3 & {[\operatorname{arcsec}]} \\
\Delta \delta_{\mathrm{M}}=26 \cos (\rho-100)-4 & {[\operatorname{arcsec}]}
\end{array}
$$

where $\rho$ is the median of the roll angle values during the considered observation expressed in degrees.

These best fits are plotted as solid lines in Fig. 2. In Table 2 we report the corresponding reduced $\chi^{2}$ values, the parameter best fit values and the associated errors at the $90 \%$ confidence level for three interesting parameters.

From the definition of $\Delta \alpha$ and $\Delta \delta$ it follows that the corrected values of LECS and MECS coordinates are given by:

$\alpha_{\mathrm{L}, \mathrm{M}}^{\mathrm{cor}}=\alpha_{\mathrm{L}, \mathrm{M}}+\Delta \alpha_{\mathrm{L}, \mathrm{M}} / \cos \delta$

$\delta_{\mathrm{L}, \mathrm{M}}^{\mathrm{cor}}=\delta_{\mathrm{L}, \mathrm{M}}+\Delta \delta_{\mathrm{L}, \mathrm{M}}$. 
Table 2. Reduced $\chi^{2}$ and best fit parameters values (the associated errors are at the $90 \%$ confidence level for three interesting parameters) relative to the best fits of Eqs. (3-6).

\begin{tabular}{lcccr}
\hline \hline Data Set & $\chi_{v}^{2}$ (d.o.f.) & $A\left[{ }^{\prime \prime}\right]$ & $\phi\left[^{\circ}\right]$ & $c\left[^{\prime \prime}\right]$ \\
\hline$\Delta \alpha_{\mathrm{L}}$ & $1.43(69)$ & $15 \pm 3$ & $-140 \pm 11$ & $2 \pm 2$ \\
$\Delta \delta_{\mathrm{L}}$ & $1.06(69)$ & $19 \pm 3$ & $-236 \pm 12$ & $-4 \pm 2$ \\
$\Delta \alpha_{\mathrm{M}}$ & $1.05(69)$ & $27 \pm 3$ & $-12 \pm 5$ & $3 \pm 2$ \\
$\Delta \delta_{\mathrm{M}}$ & $1.34(69)$ & $26 \pm 2$ & $-100 \pm 6$ & $-4 \pm 2$ \\
\hline
\end{tabular}

Equations (7) and (8) can be used to correct the sky positions derived from LECS and MECS images. Alternatively these formulae can be applied to modify the headers' keywords of LECS and MECS FITS event files (see Sect. 8).

In order to estimate the positioning accuracy of the two instruments after the coordinate correction, we have applied Eqs. (7) and (8) to the coordinates of the 72 X-ray detections of our sample and computed again the offsets between LECS/MECS and optical positions.

The results are shown in Fig. 1 (panels $b$ and d). As can be seen, the offsets are significantly reduced and the non uniform distribution of $\Delta \alpha$ and $\Delta \delta$ values for the MECS is no longer present.

The $68 \%$ and $90 \%$ error radius values for LECS and MECS after the coordinate correction are reported in Table 1 (bottom), where we can see that their positioning accuracy has improved by a factor of $\sim 2$.

The residual error in the location of X-ray sources (16" and $17^{\prime \prime}$, for LECS and MECS respectively, at the $90 \%$ confidence level) can be ascribed to the statistical uncertainty due to the Point Spread Function of the instruments (Fiore et al. 2001). Moreover, we outline that these values are of the same order (or even better) of the original LECS and MECS pixel size, showing that actually we have reached a positioning accuracy which is close to the limit of the detectors.

\subsection{Off-axis sources}

We have seen that LECS and MECS images are shifted in RA and Dec by an amount of $\sim 20^{\prime \prime}$.

Our analysis is based on the positions of on-axis sources and therefore it is not capable to check if a further rotation of the X-ray images around the center of the field of view is present. However, as the following argument shows, the effect on the coordinates of off-axis sources is expected to be negligible.

The amplitude of the rotation should be $\sim 30^{\prime \prime}$, i.e. the amount of the spacecraft attitude inaccuracy. A $30^{\prime \prime}$ rotation of the image around its center has the effect of shifting the coordinates of a source located at an off-axis angle of 30 arcmin of a very small distance $\left(\sim 0.3^{\prime \prime}\right)$ and can be therefore neglected.

We have verified this comparing the BeppoSAX X-ray and optical positions of a small sample of off-axis sources. In all cases we have found that Eqs. (7) and (8) correct properly the positions of the off-axis sources.
Table 3. LECS and MECS (three units) $68 \%$ and $90 \%$ error radius values before and after the coordinate correction of Eqs. (7) and (8) for observations carried out in the period June 1996-May 1997.

\begin{tabular}{cccc}
\hline \hline & Instrument & $68 \%$ radius & $90 \%$ radius \\
\hline before & LECS & $30^{\prime \prime}$ & $41^{\prime \prime}$ \\
correction & MECS & $37^{\prime \prime}$ & $53^{\prime \prime}$ \\
\hline after & LECS & $21^{\prime \prime}$ & $29^{\prime \prime}$ \\
correction & MECS & $19^{\prime \prime}$ & $38^{\prime \prime}$ \\
\hline
\end{tabular}

\section{Positioning accuracy before May 1998}

In this section we discuss the positioning accuracy of LECS and MECS for observations carried out before May 6th 1998.

We restricted first our study to a sample of 77 X-ray fields relative to the time interval June 1996-May 1997. The sample consists of 50 pointings of AGNs, 21 of X-ray binaries and 6 of Stars, and has been selected according to the first three criteria listed in Sect. 2. For the LECS we considered a subsample of 57 images, since in 20 of the selected fields the exposures were very short or there was a lack of data. Since the MECS1 unit was still working during this period we used coadded MECS1+MECS2+MECS3 images.

We repeated for LECS and MECS fields the analysis described in the previous sections. We found a larger scatter of the offsets between the X-ray and optical positions with respect to observations performed after May 6th 1998, as expected from the fact that the spacecraft attitude data for this sample are less accurate.

In Table 3 (top) we report the corresponding LECS and MECS $68 \%$ and $90 \%$ error radius values (numbers have been rounded to unity).

We next verified that Eqs. (3), (4), (5) and (6) describe well the dependence of $\Delta \alpha$ and $\Delta \delta$ on the roll angle and can be therefore used to correct the LECS and MECS celestial coordinates also for observations carried out during this time interval (the corresponding reduced $\chi^{2}$ values are $\chi_{v}^{2}=1.69$ for $\Delta \alpha_{\mathrm{L}}$, $\chi_{v}^{2}=1.78$ for $\Delta \delta_{\mathrm{L}}, \chi_{v}^{2}=1.42$ for $\Delta \alpha_{\mathrm{M}}$ and $\chi_{v}^{2}=1.58$ for $\Delta \delta_{\mathrm{M}}$ ).

The separate analysis of MECS1, MECS 2 and MECS 3 images confirmed that, also for the observations of this sample, there is no difference in the misalignment of the single units.

In Table 3 (bottom) we list the LECS and MECS $68 \%$ and $90 \%$ error radius values after the coordinate correction of Eqs. (7) and (8) for the 77 (57 for the LECS) X-ray detections of the sample.

As can be seen, the correction improves the source location accuracy of both instruments. Moreover, from a comparison of the error radii of Table 3 with those of Table 1, we see that the inaccurate attitude data during this period affect significantly the positioning precision of LECS and MECS.

We have not repeated the whole analysis for observations performed between June 1997 and May 6th 1998, since the rather large spacecraft attitude inaccuracies (up to 1-2 arcmin) during this time interval dominate the $\sim 20^{\prime \prime}$ residual misalignment of LECS and MECS.

Given the attitude inaccuracy, a $90 \%$ error radius of $\sim 1.2$ arcmin should be used for LECS and MECS observations 
performed during this period. A boresight correction with respect to the known position of a source in the field of view (if available) may be used to greatly reduce this error.

\section{Correction of LECS and MECS event files}

Equations (7) and (8) correct the sky positions derived from BeppoSAX LECS and MECS data. In particular the accuracy of all the celestial coordinates already published in literature can be improved applying the two formulae.

These equations can also be used to modify the headers of LECS and MECS FITS event files, thus allowing to derive from data sky positions that have the coordinate correction already applied.

To this end, we have developed a specific task that updates according to Eqs. (7) and (8) the values of the sky coordinates at reference pixel stored in the headers of LECS and MECS event files.

The task, named saxposcor, can be downloaded from a dedicated web page where a detailed description of the task and of its usage can be found (http://www.asdc.asi.it/ bepposax/coord_correction.html).

The headers of all LECS and MECS event files in the BeppoSAX archive at the ASDC have been modified with saxposcor on March 122002 and are available on-line. The BeppoSAX data reduction software SAXDAS (since version 2.3.0) includes the coordinate correction discussed here and therefore automatically corrects the headers of LECS and MECS event files.

All the details concerning the the BeppoSAX ASDC archive correction can be found on the mentioned web page.

\section{Summary}

We have presented a study of the positioning accuracy of the BeppoSAX LECS and MECS instruments using a set of archival images for which the position of the target is known to within $1^{\prime \prime}$.

We have found that a residual misalignment with respect to the spacecraft $Z$ axis of $\sim 17^{\prime \prime}$ for the LECS and $\sim 27^{\prime \prime}$ for the MECS was present.

This residual misalignment introduces a systematic error in the derived LECS and MECS source positions which depends on the spacecraft roll angle.

We give analytical formulae that can be used to correct the celestial coordinates derived from the two instruments. After the coordinate correction the source location error of LECS and MECS reduces respectively to $16^{\prime \prime}$ and $17^{\prime \prime}$ at the $90 \%$ confidence level. This represents an improvement by a factor of $\sim 2$. The residual uncertainty can be ascribed to the instrumental Point Spread Function and is of the order of the detectors pixel size.

Our work constitutes the first joint presentation of LECS and MECS imaging capabilities. The improvement presented here, which can be considered as a final reference of the location accuracy of LECS and MECS, can be useful for multiwavelength studies and future works based on BeppoSAX source catalogs.
Acknowledgements. We thank F. Fiore and P. Giommi for useful discussions and a careful supervision of the work, Tim Oosterbroek for having stimulated the beginning of this study and the referee, L. Chiappetti, for helpful comments which improved the paper. We also thank B. Saija and F. Tamburelli for their help in the development of the script saxposcor.

\section{Appendix A: On satellite attitude}

In this Appendix we give some definitions and formulae concerning the BeppoSAX attitude data, the instruments misalignment and the conversion of detector coordinates to celestial coordinates.

\section{A.1. Spacecraft attitude}

The satellite attitude is defined as the orientation of the satellite axes $(X, Y, Z)$ as a function of time.

The attitude is controlled through three on-board optical star-trackers which, for a given pointing, image the available guide stars in their field of view. When the guide stars are unavailable (e.g. for Earth occultation) the satellite orientation is determined by a set of additional instruments, i.e. by on-board gyroscopes, a solar sensor and a magnetic field sensor.

The guide stars celestial coordinates and the misalignment matrices of the three star-trackers with respect to the spacecraft axes are subsequently used on ground, by the BeppoSAX Attitude and Orbit Control Subsystem (AOCS), to reconstruct the spacecraft attitude.

Specifically, the attitude data consist of a file containing RA and Dec values of the three spacecraft axes $\left(\alpha_{X, Y, Z}\right.$ and $\left.\delta_{X, Y, Z}\right)$ as a function of time (the time bin is $0.5 \mathrm{~s}$ ).

The satellite attitude may be also described in terms of a rotation with respect to the celestial coordinate system. Following the Euler angles definitions (Goldstein 1980) we obtain the following expressions for the three rotation angles $\Phi, \Theta$ e $\Psi$ :

$\Phi=90+\alpha_{Z}$

$\Theta=90-\delta_{Z}$

$\Psi=\operatorname{atan}\left(\sin \delta_{X} / \sin \delta_{Y}\right)$.

Given the attitude Euler angles it is useful to define the roll angle $\rho$ :

$\rho=\Psi-90$

and to describe the spacecraft attitude with the three angles $\alpha_{Z}$, $\delta_{Z}$ and $\rho$.

\section{A.2. Instruments misalignments}

Given a satellite attitude reconstruction, in order to associate a celestial coordinates grid to the LECS and MECS images the misalignment between the optical axes of the X-ray telescopes with respect to the satellite axes $(X, Y, Z)$ has to be taken into account.

This misalignment is described in terms of three subsequent rotations around the satellite axes. If we call $M_{\text {mis }}$ the instruments misalignment matrix and $M_{\text {att }}$ the spacecraft Euler 
matrix associated to the angles $\Phi, \Theta$ e $\Psi$, we can define the matrix $M_{\text {det }}$

$M_{\mathrm{det}}=M_{\mathrm{mis}} M_{\mathrm{att}}$

which allows to associate a celestial coordinate system to the detector image. The misalignment matrices $M_{\text {mis }}$ have been computed by the LECS and MECS scientific teams.

\section{A.3. Sky coordinates computation}

A detailed discussion of the procedure used to convert LECS and MECS raw detector pixels to linearized detector pixels and then to sky pixels is beyond the scope of this Appendix and we briefly summarise only the main steps.

First, the raw (electronic) detector pixels are converted to a physical distance (in $\mathrm{mm}$ ) on the detector surface. Since the response of the detector is affected by some nonlinearities, which have been calibrated on ground, this step involves a coordinate linearization which corrects image distortions.

Second, the distance in $\mathrm{mm}$ on the detector is converted to detector linearized pixels. A choice of a pixel size of $8^{\prime \prime}$, for both LECS and MECS, is included in this step.

Third, detector linearized pixels are converted to sky coordinates pixels. This step involves the use of the matrix $M_{\mathrm{det}}$ which allows to associate celestial coordinates to the detector linearized image.

The celestial coordinates of a "reference" detector pixel and the roll angle, computed as the median of the values assumed during the observation, are stored in the headers of LECS and MECS event files.

\section{References}

Antonelli, L. A., Piro, L., Vietri, M., et al. 2000, ApJ, 545, 39

Boella, G., Butler, R. C., Perola, G. C., et al. 1997a, A\&AS, 122, 299

Boella, G., Chiappetti, L., Conti, G., et al. 1997b, A\&AS, 122, 327

Comastri, A., Fiore, F., Vignali, C., et al. 2001, MNRAS, 327, 781

Feroci, M., Antonelli, L. A., Soffitta, P., et al. 2001, A\&A, 378, 441

Fiore, F., La Franca, F., Giommi, P., et al. 1999, MNRAS, 306, 55

Fiore, F., Giommi, P., Vignali, C., et al. 2001, MNRAS, 327, 771

Giommi, P., Angelini, L., Jacobs, P., \& Tagliaferri, G. 1992, ASP Conf. Ser., 25, 100

Giommi, P., \& Fiore, F. 1997, Data Analysis in Astronomy IV, 93

Giommi, P., Fiore, F., Ricci, D., et al. 1998, Nucl. Phys. B, 69, 691

Giommi, P., Perri, M., \& Fiore, F. 2000, A\&A, 362, 799

Goldstein, H. 1980, Classical Mechanics, Addison-Wesley

in 't Zand, J. J. M., Kuiper, L., Amati, L., et al. 2001, ApJ, 559, 710

in 't Zand, J. J. M., Miller, J. M., Oosterbroek, T., \& Parmar, A. N. 2002, A\&A, 394, 553

La Franca, F., Fiore, F., Vignali, C., et al. 2002, ApJ, 570, 100

Matteuzzi, A. 1998, Ph.D. Thesis, Padova University

Parmar, A. N., Martin, D. D. E., Bavdaz, M., et al. 1997, A\&AS, 122, 309

Piro, L., Frail, D. A., Gorosabel, J., et al. 2002, ApJ, in press [astro-ph/0201282]

Ricci, D., Fiore, F., \& Giommi, P. 1998, Nucl. Phys. B, 69, 618

Turon, C., Creze, M., Egret, D., et al. 1992, ESA SP-1136

van Paradijs, J. 1995, X-ray Binaries, ed. W. H. G. Lewin, J. van Paradijs, \& E. P. J. van den Heuvel (Cambridge University Press)

Veron-Cetty, M.-P., \& Veron, P. 1996, A\&AS, 115, 97

Vignali, C., Comastri, A., Fiore, F., \& La Franca, F. 2001, A\&A, 370, 900 